\title{
IRAS 09425-6040: A carbon star surrounded by highly crystalline silicate dust $^{\star, \star \star}$
}

\author{
F. J. Molster ${ }^{1, \dagger}$, I. Yamamura ${ }^{1,2}$, L. B. F .M. Waters ${ }^{1,3}$, L.-Å Nyman ${ }^{4,5}$, H.-U. Käufl ${ }^{4}$, \\ T. de Jong ${ }^{1,6}$, and C. Loup ${ }^{7}$
}

1 Astronomical Institute "Anton Pannekoek", University of Amsterdam, Kruislaan 403, 1098 SJ Amsterdam, The Netherlands

2 The Institute of Space and Astronautical Science, Yoshino-dai 3-1-1, Sagamihara, Kanagawa 229-8510, Japan

3 Instituut voor Sterrenkunde, Katholieke Universiteit Leuven, Celestijnenlaan 200B, 3001 Heverlee, Belgium

4 European Southern Observatory, Casilla 19001, Santiago 19, Chile

5 Onsala Space Observatory, 43992 Onsala, Sweden

6 SRON Laboratory for Space Research, Sorbonnelaan 2, 3584 CA Utrecht, The Netherlands

7 Institut d'Astrophysique de Paris, 98bis Boulevard Arago, 75014 Paris, France

Received 14 June 2000 / Accepted 16 November 2000

\begin{abstract}
We present infrared spectroscopy and millimeter photometry and spectroscopy of the peculiar carbon star IRAS 09425-6040. The 2-15 $\mu \mathrm{m}$ spectrum, as well as the CO millimeter line observations are typical for a (J-type) carbon star with moderate mass-loss rate. The $15-45 \mu \mathrm{m}$ spectrum is dominated by strong emission bands from Mg-rich and Fe-poor crystalline silicates. IRAS 09425-6040 has the highest abundance of crystalline silicates (75 per cent) observed in any source so far. The ISO data, combined with IRAS and millimeter wavelength photometry indicate the presence of large cold grains. The observations indicate that the carbon star IRAS 09425-6040 is surrounded by a stationary, massive, highly crystalline oxygen-rich dust disk which is depleted of gas. These properties are very similar to those of the disk seen in the Red Rectangle. We propose that IRAS 09425-6040 is the evolutionary progenitor of the central binary of the Red Rectangle nebula.
\end{abstract}

Key words. infrared: stars - stars: AGB and post-AGB; mass loss - planetary nebulae: general - dust, extinction

\section{Introduction}

The evolution of low- and intermediate-mass stars ends with a phase of very high mass loss when they are cool giants on the Asymptotic Giant Branch (AGB). In the extended outer layers of the stellar atmosphere, molecules can condense into solid material (dust). These dust grains can efficiently absorb stellar radiation which heats them to temperatures up to $1000-1500 \mathrm{~K}$. In addition, the radiation pressure exerted on the grains causes an outward acceleration, i.e. the dust grains are important for the dynamics of the flow and the mass loss.

Send offprint requests to: F. J. Molster,

e-mail: fjmolster@mvainc.com

$\dagger$ Current address: MVA Inc, 5500 Oakbrook Parkway suite 200, Norcross, GA 30093, USA.

* Based on observations with ISO, an ESA project with instruments funded by ESA Member States (especially the PI countries: France Germany, The Netherlands and the UK) and with the participation of ISAS and NASA.

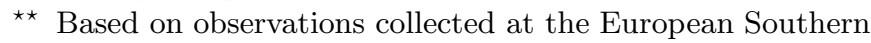
Observatory, la Silla, Chili.
The chemistry of the dust which condenses in AGB winds is determined by the $\mathrm{C} / \mathrm{O}$ ratio, which initially is less than unity, resulting in the formation of oxygen-rich dust (e.g. silicates and simple oxides like $\mathrm{Al}_{2} \mathrm{O}_{3}$ ). Due to the production of excess carbon in the nuclear burning shells that surround the degenerate $\mathrm{C} / \mathrm{O}$ core, and its subsequent dredge-up to the surface, the $\mathrm{C} / \mathrm{O}$ ratio gradually approaches, and eventually exceeds unity, resulting in the formation of a carbon star. The dust in the winds of carbon stars mostly consists of amorphous carbon, $\mathrm{SiC}$, and possibly MgS.

In single AGB stars, the material in the wind, once removed from the gravitational potential well of the star, can freely escape into the interstellar medium. In binary systems, however, some of the material can be trapped and stored in the system for longer periods of time. Depending on the orbital separation and nature of the companion, matter may be stored in a disk around the companion or around the entire system (see e.g. Yamamura et al. 2000). A well-known example of the latter case is the central star of the Red Rectangle nebula, HD 44179 (Waelkens et al. 1996; Osterbart et al. 1997). This object has recently left 

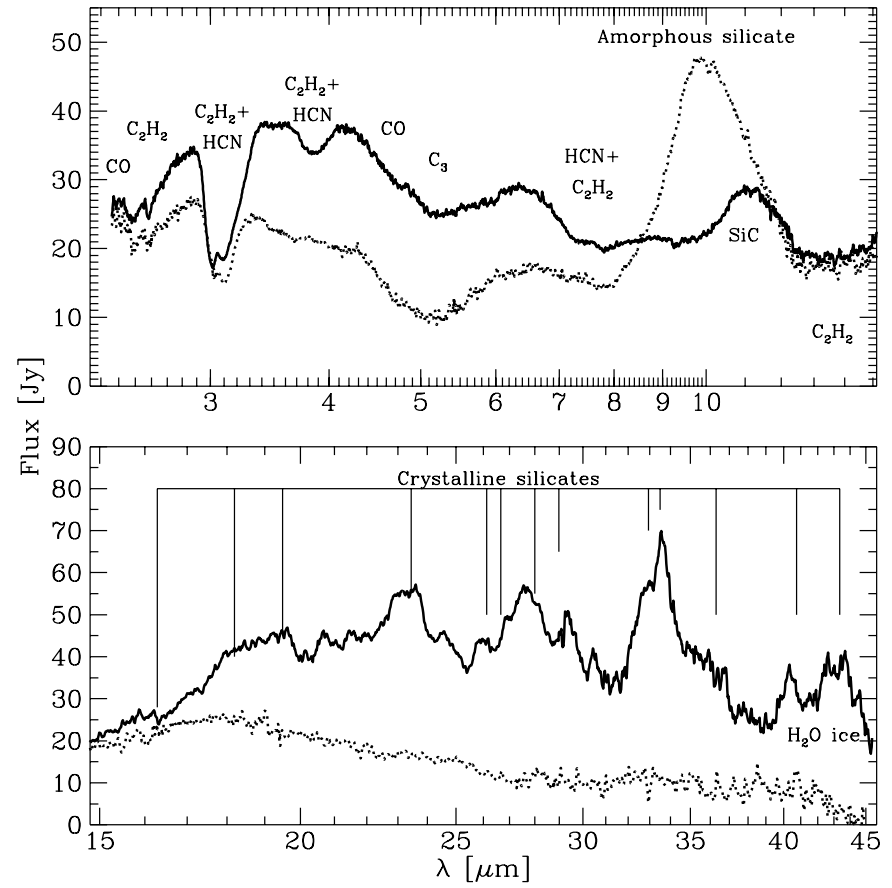

Fig. 1. The SWS spectrum of IRAS 09425-6040 (solid line), compared with the spectrum of a typical silicate carbon star V778 Cyg (dotted line). The spectral features are indicated in the figure

the AGB and is rapidly evolving to higher $T_{\text {eff }}$. The Red Rectangle has a pronounced carbon-rich dust shell, but surprisingly also a prominent oxygen-rich dust component (Waters et al. 1998). The mixed chemistry can be understood in the context of binary AGB evolution, in which a reservoir of oxygen-rich dust is stored in the system, and the star subsequently evolves to the carbon-rich phase.

In this paper we report the discovery of a silicate carbon star with a massive and highly crystalline oxygen-rich dust shell. Based on its IRAS colours, IRAS 09425-6040 was classified as a post-AGB object and selected for observations in the guaranteed time of the Short-Wavelength Spectrometer (SWS, de Graauw et al. 1996) on board of the Infrared Space Observatory (ISO, Kessler et al. 1996). The ISO-SWS spectrum however revealed the presence of a carbon-rich AGB star surrounded by a highly crystalline silicate dust shell. The paper is organized as follows: in Sect. 2, we describe the infrared and sub-millimeter observations of IRAS 09425-6040. Section 3 describes modelling of the observations, and in Sect. 4 we discuss the nature and evolutionary status of IRAS 09425-6040.

\section{The observations}

IRAS 09425-6040 was observed on 27 July, 1996 using ISO-SWS as part of the guaranteed time observing programme. A full scan with SWS was obtained (AOT01 speed 4) covering the wavelength range between 2.38 and $45.2 \mu \mathrm{m}$. The data were reduced using the SWS Interactive Analysis package version 7.0. The final spectrum was obtained by averaging the spectra of the 12 individual de- tectors, after removing outlying points by sigma-clipping and eye inspection. The spectrum is shown in Fig. 1.

The short wavelength part $(2.38-15 \mu \mathrm{m})$ of the spectrum is dominated by deep absorption bands from carbonrich gas-phase molecules that are located in the atmosphere of the star, and in warm circumstellar layers, i.e. the innermost layer of the circumstellar envelope. We identify $\mathrm{C}_{2} \mathrm{H}_{2}, \mathrm{HCN}, \mathrm{CO}$ and $\mathrm{C}_{3}$ (Yamamura et al. 1998). At 10-12 $\mu \mathrm{m}$, emission from warm $\mathrm{SiC}$ dust grains is evident, with a peak at $11.3 \mu \mathrm{m}$. We conclude that IRAS 09425-6040 is a carbon-rich AGB star with a moderate mass-loss rate. In Fig. 1 we also show the SWS spectrum of the carbon star V778 Cyg (Yamamura et al. 2000). The 2-8 $\mu \mathrm{m}$ part of the spectrum of IRAS $09425-6040$ is very similar to that of V778 Cyg, and is typical for normal carbon-rich red giants and those with amorphous silicate dust shells.

In contrast, the $15-45.2 \mu \mathrm{m}$ part of the spectrum of IRAS $09425-6040$ is dominated by strong, narrow solid state emission bands from oxygen-rich crystalline silicates, as evidenced by peaks near $16.5,19.5,23.8,27.5,33.8$ and $36.5 \mu \mathrm{m}$ due to crystalline olivines $\left(\mathrm{Mg}_{x} \mathrm{Fe}_{1-x}\right)_{2} \mathrm{SiO}_{4}$ $[0 \leq x \leq 1]$, and peaks near 18.2, 23.2, 28.0, 36.0, 40.5, and $43.8 \mu \mathrm{m}$ due to crystalline pyroxenes $\left(\mathrm{Mg}_{x} \mathrm{Fe}_{1-x}\right) \mathrm{SiO}_{3}$ $[0 \leq x \leq 1]$ (Koike \& Shibai 1998; Jäger et al. 1998). These bands are absent in V778 Cyg, indicating a large difference in ratio between amorphous and crystalline silicates between both stars. In Table 1 we list the strongest bands with their central wavelength and their identification.

The remarkable mix of both oxygen-rich and carbonrich components may be due to a chance superposition of two IR sources in the SWS beam. We observed IRAS 09425-6040 with the TIMMI $10 \mu \mathrm{m}$ camera attached to the ESO $3.6 \mathrm{M}$ camera in the broad-band $N$ filter (spatial resolution $<1^{\prime \prime}$ ) and found that only one bright IR source is in the SWS aperture. We conclude that the oxygen-rich and carbon-rich components are physically associated.

The infrared continuum flux level is roughly constant between 15 and $45 \mu \mathrm{m}$ at about $20 \mathrm{Jy}$. The IRAS $60 \mu \mathrm{m}$ broad-band flux of 21.2 Jy suggests that the continuum remains flat to at least that wavelength. This is not typical for a detached dust shell with $r^{-2}$ density gradient, but suggests a much flatter density gradient.

We have observed IRAS 09425-6040 with the Swedish ESO Sub-millimeter Telescope (SEST) at the European Southern Observatory (ESO) using the bolometer at a wavelength of $1.3 \mathrm{~mm}$. It was detected at a continuum flux level of $14.4 \pm 3.4$ mJy. In addition, we detected emission in the pure rotational transitions of ${ }^{12} \mathrm{CO}$ at $J=1-0$, $J=2-1$ and of ${ }^{13} \mathrm{CO}$ at $J=2-1$ at frequencies of 115,230 and $220 \mathrm{GHz}$ respectively (see Fig. 2). All three $\mathrm{CO}$ emission profiles peak at the same velocity of about $+15 \pm 3 \mathrm{~km} \mathrm{~s}^{-1}$, indicating that we are looking at the same gas in all three cases. The shape of the lines is consistent with an origin in the present-day outflow of the carbon star. 
Table 1. The results of the Gauss-fits to the emission bands in the infrared spectrum of IRAS 09425-6040 and the identifications. A "?" denotes uncertain identifications

\begin{tabular}{|c|c|c|c|c|}
\hline $\begin{array}{c}\lambda \\
\mu \mathrm{m}\end{array}$ & $\begin{array}{c}F W H M \\
\mu \mathrm{m}\end{array}$ & $I_{\text {peak }} / I_{\text {cont }}$ & $\begin{array}{c}F_{\text {band }} \\
10^{-14} \mathrm{Wm}^{-2}\end{array}$ & Identifications \\
\hline $10.64 \pm .03$ & $.49 \pm .32$ & $1.066 \pm .044$ & $2.7 \pm 3$ & enstatite? \\
\hline $11.13 \pm .07$ & $1.31 \pm .08$ & $1.386 \pm .039$ & $27 \pm 4$ & $\mathrm{SiC}$ \\
\hline $12.02 \pm .02$ & $.47 \pm .03$ & $1.094 \pm .011$ & $1.9 \pm .2$ & \\
\hline $15.13 \pm .01$ & $.29 \pm .01$ & $1.055 \pm .001$ & $.46 \pm .02$ & enstatite \\
\hline $15.79 \pm .01$ & $.65 \pm .02$ & $1.134 \pm .006$ & $2.5 \pm .2$ & silica? \\
\hline $16.19 \pm .01$ & $.10 \pm .01$ & $1.079 \pm .003$ & $.23 \pm .01$ & forsterite \\
\hline $16.97 \pm .02$ & $.52 \pm .06$ & $1.104 \pm .012$ & $1.6 \pm .3$ & \\
\hline $18.09 \pm .01$ & $1.24 \pm .05$ & $1.324 \pm .017$ & $12 \pm .9$ & enstatite + forsterite \\
\hline $19.17 \pm .01$ & $.94 \pm .04$ & $1.223 \pm .010$ & $6.5 \pm .6$ & forsterite? \\
\hline $19.63 \pm .01$ & $.30 \pm .01$ & $1.116 \pm .003$ & $1.1 \pm .05$ & forsterite + enstatite \\
\hline $20.69 \pm .01$ & $.37 \pm .05$ & $1.135 \pm .036$ & $1.5 \pm .5$ & silica? diopside? \\
\hline $21.49 \pm .01$ & $.27 \pm .01$ & $1.059 \pm .002$ & $.48 \pm .03$ & \\
\hline $22.43 \pm .01$ & $.22 \pm .01$ & $1.032 \pm .003$ & $.20 \pm .03$ & \\
\hline $23.03 \pm .01$ & $.72 \pm .01$ & $1.269 \pm .017$ & $5.1 \pm .3$ & enstatite \\
\hline $23.65 \pm .01$ & $.54 \pm .02$ & $1.283 \pm .019$ & $3.7 \pm .3$ & forsterite \\
\hline $24.57 \pm .03$ & $.67 \pm .05$ & $1.132 \pm .022$ & $1.9 \pm .3$ & enstatite \\
\hline $25.07 \pm .02$ & $.25 \pm .04$ & $1.034 \pm .002$ & $.16 \pm .03$ & diopside? \\
\hline $25.99 \pm .02$ & $.62 \pm .04$ & $1.191 \pm .017$ & $2.1 \pm .3$ & forsterite + silica? \\
\hline $26.82 \pm .01$ & $.40 \pm .14$ & $1.174 \pm .077$ & $1.3 \pm .9$ & \\
\hline $27.56 \pm .08$ & $1.18 \pm .39$ & $1.537 \pm .091$ & $9.8 \pm 4$ & forsterite \\
\hline $28.28 \pm .12$ & $.76 \pm .19$ & $1.207 \pm .168$ & $2.7 \pm 2$ & enstatite \\
\hline $29.39 \pm .02$ & $.74 \pm .13$ & $1.348 \pm .099$ & $3.5 \pm 1$ & diopside? \\
\hline $30.48 \pm .01$ & $.32 \pm .07$ & $1.190 \pm .041$ & $.75 \pm .3$ & \\
\hline $32.16 \pm .08$ & $.32 \pm .17$ & $1.154 \pm .082$ & $.61 \pm .7$ & diopside? \\
\hline $32.84 \pm .03$ & $1.00 \pm .14$ & $1.705 \pm .039$ & $6.9 \pm 1$ & \\
\hline $33.63 \pm .01$ & $.63 \pm .02$ & $1.950 \pm .028$ & $5.5 \pm .4$ & forsterite \\
\hline $34.19 \pm .01$ & $.55 \pm .07$ & $1.344 \pm .072$ & $1.7 \pm .5$ & diopside? \\
\hline $35.02 \pm .10$ & $1.42 \pm .39$ & $1.358 \pm .021$ & $4.0 \pm 1$ & clino-enstatite? \\
\hline $35.98 \pm .03$ & $.73 \pm .26$ & $1.275 \pm .032$ & $1.5 \pm .7$ & ortho-enstatite? \\
\hline $36.72 \pm .04$ & $.37 \pm .09$ & $1.268 \pm .046$ & $.67 \pm .2$ & forsterite+ ? \\
\hline $39.70 \pm .02$ & $.21 \pm .05$ & $1.136 \pm .011$ & $.13 \pm .03$ & diopside? \\
\hline $40.35 \pm .01$ & $.93 \pm .06$ & $1.607 \pm .073$ & $2.5 \pm .3$ & enstatite $+?$ \\
\hline $41.53 \pm .05$ & $.92 \pm .19$ & $1.335 \pm .100$ & $1.2 \pm .5$ & 41 micron plateau \\
\hline $42.55 \pm .06$ & $.85 \pm .18$ & $1.629 \pm .167$ & $2.1 \pm .9$ & cryst. $\mathrm{H}_{2} \mathrm{O}$-ice \\
\hline $43.60 \pm .08$ & $1.30 \pm .50$ & $1.883 \pm .134$ & $.4 .0 \pm 2$ & ortho-enstatite \\
\hline $44.58 \pm .01$ & $.44 \pm .02$ & $1.460 \pm .023$ & $.69 \pm .04$ & clino-enstatite, diopside? \\
\hline
\end{tabular}

\section{Modelling}

We have modelled the SWS-spectrum from $15-45.2 \mu \mathrm{m}$ together with the IRAS 60 and $100 \mu \mathrm{m}$ points and the SEST $1.3 \mathrm{~mm}$ continuum point, using a spherically symmetric radiative transfer model (MODUST, Bouwman \& de Koter, in preparation).

We assumed a luminosity of $6.510^{3} L_{\odot}$ and temperature of $2500 \mathrm{~K}$, typical for carbon-rich red giants (Groenewegen et al. 1995). A distance of $1.3 \mathrm{kpc}$ was derived based on this luminosity.

We assumed that the grain size distribution could be represented by a single-size grain population. We were not able to model the spectrum of IRAS 09425-6040 with grains smaller than $2 \mu \mathrm{m}$. The 10 micron silicate feature would become too strong and would have been seen together with the $\mathrm{SiC}$ feature. Even for $2 \mu \mathrm{m}$ sized grains the silicate feature is still rather strong, which suggests that larger grains might produce an even better fit in that region. However, this would give problems with the crystalline silicates at the longer wavelengths. We preferred to use the same grain size for the amorphous and the crystalline silicates and therefore we adopted a grain size of $2 \mu \mathrm{m}$.

Above $15 \mu \mathrm{m}$ the emission from the carbon-rich dust in the present-day wind of the carbon star is negligible. The published optical constants of the crystalline silicates are not able to reproduce the correct peak positions as observed in the laboratory and in the ISO-data (Molster et al., in preparation). We therefore decided to take the extinction coefficients $(Q)$ derived from laboratory measurements by Koike et al. (1999) for enstatite and forsterite. A comparison with the ISO-data of stars with crystalline silicates showed that this dataset reproduces the observed peaks very well (Molster et al., in preparation). To extend these datasets to shorter and longer wavelengths we used the optical constants of amorphous enstatite and forsterite 


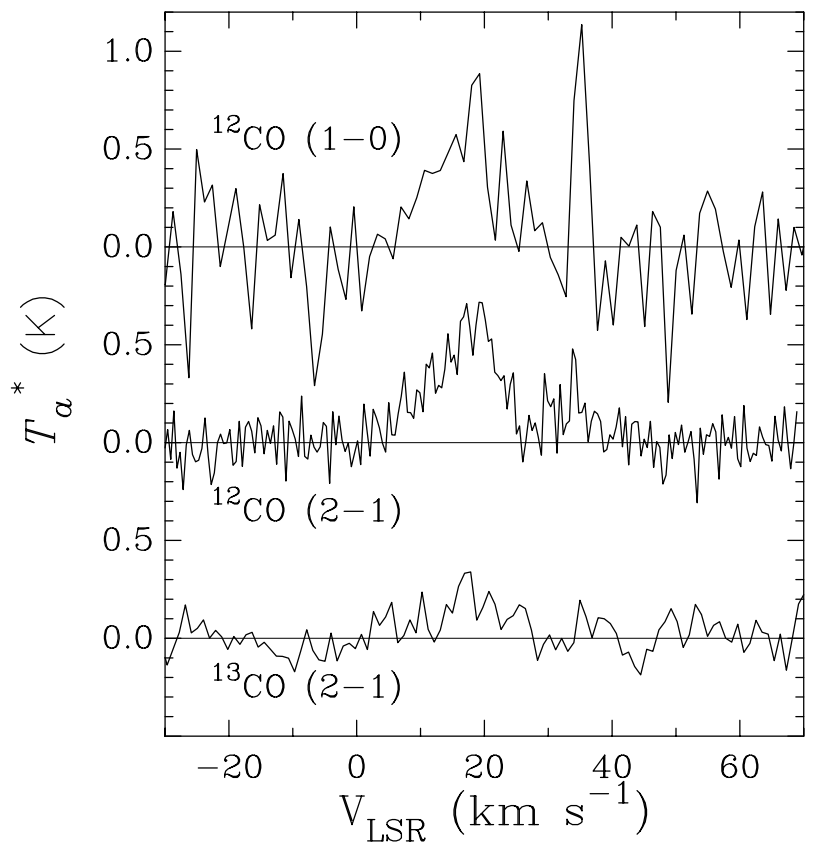

Fig. 2. The observed CO pure-rotational lines in IRAS 09425-6040.

Table 2. The results of our model fit to the spectrum of IRAS 09425-6040

\begin{tabular}{|l|c|c|c|l|}
\hline Dust component & $\begin{array}{c}\text { size } \\
(\mu \mathrm{m})\end{array}$ & $\begin{array}{c}T \text { range } \\
(\mathrm{K})\end{array}$ & $\begin{array}{c}\text { Mass } \\
10^{-4} M_{\odot}\end{array}$ & Ref. \\
\hline Amorph. silicates & 2.0 & $220-43$ & 3.2 & $\mathrm{a}, \mathrm{b}, \mathrm{c}$ \\
Forsterite & 2.0 & $148-36$ & 2.1 & $\mathrm{~d}, \mathrm{e}$ \\
Clino-enstatite & 2.0 & $117-27$ & 5.0 & $\mathrm{~d}, \mathrm{e}$ \\
Ortho-enstatite & 2.0 & $118-27$ & 5.0 & $\mathrm{~d}, \mathrm{e}$ \\
\hline Amorph. silicates & 300. & $223-20$ & 5.7 & $\mathrm{a}, \mathrm{b}, \mathrm{c}$ \\
\hline
\end{tabular}

References: $\mathrm{a}=$ Dorschner et al. (1995); b = Laor \& Draine (1993); c = Ossenkopf et al. (1992); d = Koike et al. (1999); $\mathrm{e}=$ Scott \& Duley (1996).

of Scott \& Duley (1996) and calculated the absorption coefficients for spherical particles. We assumed that the optical behaviour is not very different for amorphous and crystalline grains at these wavelengths, i.e. outside the region of the strong crystalline silicate features. A similar approach was used for the amorphous grains. We used the optical constants derived by Dorschner et al. (1995), to calculate the extinction coefficients for the amorphous olivine $\left(\mathrm{MgFeSiO}_{4}\right)$. An extension to longer and shorter wavelengths was achieved using the optical constants of Ossenkopf et al. (1992) and Laor \& Draine (1993). We calculated the dust emission for an optically thin shell. In the final fit, we found that, apart from two narrow regions around the forsterite peaks at 11.3 and $19.5 \mu \mathrm{m}, \tau$ was for all relevant wavelengths lower than 0.2 , validating our assumption.

We found a density gradient $\rho \propto r^{-1.6 \pm 0.1}$, which is significantly flatter than expected for a (detached) outflow. This value is more in line with a (stationary) disk geometry. Indeed, the flat continuum slope between 15

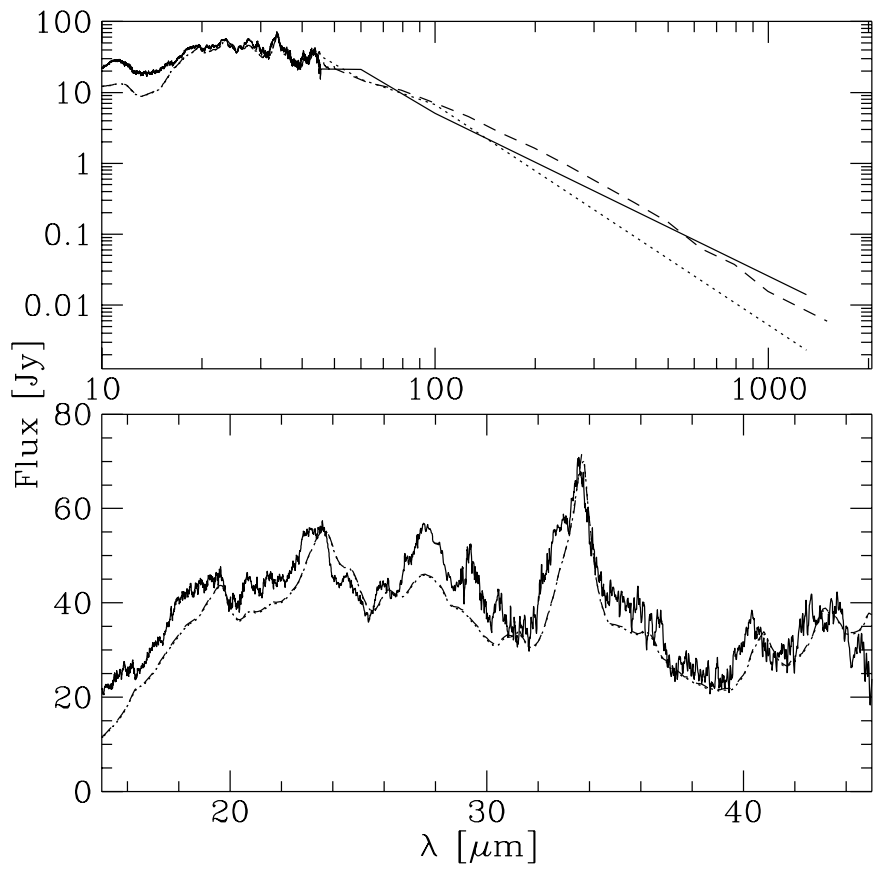

Fig. 3. The model fit (dotted line) to the dust spectrum of IRAS 09425-6040 (solid line). The dashed line gives the fit when $300 \mu \mathrm{m}$ grains are added (see Sect. 4.3). Note the similarity up to $45 \mu \mathrm{m}$

and $60 \mu \mathrm{m}$ is not consistent with an outflow origin of the cool dust, but more compatible with a disk-like structure.

In Fig. 3 we show the final fit to the spectrum, and in Table 2 we give the most important parameters of the dust species. The inner and outer radii are respectively $2.0 \pm 0.210^{15}$ and $2.3 \pm 0.610^{17} \mathrm{~cm}$ and the disk contains a total dust mass of about $1.5 \pm 0.310^{-3} M_{\odot}$. The model fits the spectrum very well in the SWS wavelength range, both on a global scale as well as several of the dust features. We will discuss the far-infrared part in Sect. 4.3.

We note that the temperature derived for the enstatite is low compared to that of other dust components (see Table 2), and it is also low compared to the colour temperature derived from the observed 28 to $43 \mu \mathrm{m}$ band strength ratio. As for all dust components, in our calculations the enstatite temperature is determined by the energy balance between absorbed and emitted radiation. Unfortunately, no reliable laboratory data are available for the opacity of crystalline enstatite at wavelengths shortwards of about $7 \mu \mathrm{m}$. For our calculations we have used the laboratory data of amorphous enstatite at $\lambda<7 \mu \mathrm{m}$. However, the opacity of amorphous and crystalline enstatite may differ at short wavelengths, and this may affect the derived crystalline enstatite temperature. We conclude that the crystalline enstatite temperature may be too low, and therefore the derived dust mass of this component may be too high.

About $60-80 \%$, depending on the temperature correction for enstatite, of the small $(2 \mu \mathrm{m})$ dust particles are 
crystalline: the highest percentage found in the whole ISO sample.

The errors in our modelling for the properties of the other dust species depend largely on the assumed size of the dust particles. This can change the derived masses by more than a factor 2. Also the temperatures at the inner radius derived in Table 2 are uncertain by about $10 \mathrm{~K}$ for the forsterite and small amorphous silicate grains.

We can estimate the gas to dust ratio in the cool dust component by analysing the CO line emission. The CO gas associated with the cool oxygen-rich dust disk is expected to produce a narrow (1-5 $\mathrm{km} \mathrm{s}^{-1}$ full width) line (e.g. Jura \& Kahane 1999), which is not found. This places an upper limit to the gas mass in the cool disk of $210^{-3} M_{\odot}$ for a $\mathrm{CO}$ to $\mathrm{H}_{2}$ abundance ratio of $310^{-4}$ (Knapp \& Morris 1985). The gas to dust mass ratio in the disk is very low, at least $<10$ (taking possible errors in the dust modelling into account), and implies significant gas depletion.

\section{Discussion}

\subsection{The underlying carbon star}

From the strength and shape of the CO pure rotational emission lines we derive an outflow velocity of $8.0 \mathrm{~km} \mathrm{~s}^{-1}$ and a mass-loss rate of $210^{-6} M_{\odot} \mathrm{yr}^{-1}$. The mass-loss rate found for $\mathrm{CO}$ is in good agreement with that derived from the strength of the $\mathrm{SiC}$ dust feature. Furthermore, a ${ }^{12} \mathrm{C}$ over ${ }^{13} \mathrm{C}$ abundance ratio of approximate 10 is found, which is low and suggests that IRAS 09425-6040 is a Jtype carbon-star, alike the rare group of carbon stars with amorphous silicate dust shells (Willems \& de Jong 1996; Lloyd Evans 1990; Lambert et al. 1990). We have searched for $\operatorname{SiO}(v=0, J=2-1)$ and $\operatorname{HCN}(J=1-0)$, but only upper limits of respectively $710^{-11}$ and $510^{-11} M_{\odot}$, were obtained.

\subsection{The cool silicate dust}

The position and strength of the peaks of the crystalline silicate emission bands in IRAS 09425-6040 suggest a very high $\mathrm{Mg} / \mathrm{Fe}$ ratio consistent with the magnesiumrich end members of the solid solution series of olivine and pyroxene, respectively forsterite $\left(\mathrm{Mg}_{2} \mathrm{SiO}_{4}\right)$ and enstatite $\left(\mathrm{MgSiO}_{3}\right)$. IRAS $09425-6040$ shows the most prominent crystalline silicate emission observed so far in any object (Molster et al. 1999a). While in the winds of oxygenrich red giants the bulk of the silicates is Fe-rich and amorphous, and the abundance of the Fe-poor crystalline dust is of the order of 10 per cent or less (Waters et al. 1996; Waters \& Molster 1999; Molster et al. 1999b), in IRAS 09425-6040 the situation is reversed: the crystalline silicates are the most dominant components in the spectrum, with only a minor contribution from amorphous silicates. The present-day silicate dust composition therefore is likely not representative of that when the silicate dust was formed in the red giant wind.
Such a high abundance of crystalline silicates is a property of long-lived, stationary disks surrounding stars in very different evolutionary phases, both young and old (Molster et al. 1999a). This again suggests that IRAS 09425-6040 contains a long-lived disk of oxygenrich material. Molster et al. (1999a) suggest several mechanisms to increase the degree of crystallinity in long-lived oxygen-rich dust disks: removal of amorphous grains, coagulation of small amorphous grains and crystallization of the grains below the glass temperature. They conclude that the latter scenario is the more likely one, although the exact mechanism (ion bombardment, UV radiation, or another unknown mechanism) is not known yet.

\subsection{The far-infrared}

Even with the flat density distribution we were not able to fit the far-IR and mm continuum points. A population of $2 \mu \mathrm{m}$ sized grains far away from the star (and thus very cold), could be excluded since they would be located outside the SEST beam $\left(23^{\prime \prime}\right.$ at $\left.1.3 \mathrm{~mm}\right)$.

The $60 \mu \mathrm{m}$ over $1.3 \mathrm{~mm}$ flux ratio is relatively low $\left(F_{\nu} \propto \nu^{2.4}\right)$. The frequency dependence of the absorption coefficient of the dust species used in our model follows, for the long wavelengths a power law $\kappa(\nu) \propto \nu^{\beta}$, with $\beta \approx 2.0$. Agladze et al. (1996) and Mennella et al. (1998) showed that $\beta$ is temperature dependent and can become lower when the temperature drops. However their results do not even come close to $\beta=0.4$ which would be necessary to explain the slope at the far infrared, assuming we are at the Rayleigh-Jeans tail of the energy distirbution.

Since we can rule out the temperature dependent $\beta$ as main cause for the gentle slope, a reasonable alternative would be the presence of a population of large (i.e. close to blackbody size), cold grains. To simulate these large grains we added $300 \mu \mathrm{m}$ sized spherical amorphous silicate grains to the dust mixture and this improves the fit (see Fig. 3 and Table 2). Their size is somewhat arbitrary, but had to be much larger than the other $2 \mu \mathrm{m}$ dust grains. This population of large grains seems indeed a distinct grain population. A reasonable size distribution $\left(n(a) \propto a^{-q} \mathrm{~d} a\right.$, with $a$ the particle size and the exponent $q$ between 2.5 and 4) will result in too much flux in the far-infrared, if the mm-continuum flux is fixed. It should be noted that the exact dust composition of these large grains is difficult to determine. The mass of the large grains will be of the same order as the mass in the "small" grains. The exact mass and temperature of the large grains depends both on the adopted grain size and optical properties (read chemical composition).

We want to note that similar gentle slopes are found in spectra of young stars and the evolved star the Red Rectangle. The presence of a population of large grains in a disk was found necessary to explain these spectra (Bouwman et al. in preparation; Jura et al. 1997). 


\subsection{The evolutionary status of IRAS 09425-6040}

The properties of the oxygen-rich dust shell surrounding IRAS 09425-6040 are very similar to those of the Red Rectangle (Waters et al. 1998). In the Red Rectangle, both carbon-rich dust (polycyclic aromatic hydrocarbons $(\mathrm{PAHs})$ ) and crystalline silicates are present. In addition, a population of large, cold grains was found in the Red Rectangle (Jura et al. 1997), as well as strong evidence for depletion of the gas in the cold (oxygen-rich) dust component (Jura et al. 1995). From IR imaging and ISO spectroscopy a geometry of the dust is suggested in which the oxygen-rich material is in the disk, and the carbonrich dust is mainly concentrated in the X-shaped nebula (Waters et al. 1998). From radial velocity studies it was shown that the Red Rectangle consists of a central binary system in a 318 day orbit (Waelkens et al. 1996). One component has evolved off the AGB and is now an F type supergiant, rapidly heating up to become a hot white dwarf; the other star in the system is not seen directly. The disk seen in direct imaging is a circum-binary disk.

We propose that IRAS $09425-6040$ is also a binary and the evolutionary progenitor to the Red Rectangle since the similarities between the disks in the Red Rectangle and in IRAS 09425-6040 are striking: both stars have a present-day carbon-rich outflow and an oxygen-rich dust disk, which is gas depleted. They have a relatively low $60 \mu \mathrm{m} / 1.3 \mathrm{~mm}$ flux ratio, which is an indication for the presence of large grains, and the oxygen-rich disk is highly crystalline. The abundance of $13 \%$ crystalline silicates in the Red Rectangle disk (Waters et al. 1998) is a lower limit due to the strong carbon-rich warm dust contribution to the continuum at longer wavelengths. The main difference between IRAS 09425-6040 and the Red Rectangle is the lack of warm (carbon-rich) dust in the disk of the former system emitting at the shortest wavelengths.

What is the relation between IRAS 09425-6040, the Red Rectangle and the silicate carbon stars with warm silicates? This question is difficult to answer without better knowledge concerning, e.g. the binary nature of the silicate carbon stars and the orbital parameters. It seems difficult to picture objects like V778 Cyg as progenitors to IRAS 09425-6040, since the amount of oxygen-rich material is much higher in IRAS 09425-6040. Note that no more oxygen-rich dust is produced as the mass-losing star is now carbon-rich. Apparently the efficiency with which oxygen-rich dust was stored in IRAS 09425-6040 and the Red Rectangle was much higher than in objects as V778 Cyg. This may be related to the nature of the companion and to the orbital parameters. In a recent study Yamamura et al. (2000) show that the formation of an oxygen-rich dust reservoir in wide binary systems likely occurs in a disk surrounding the companion (e.g. in V778 Cyg) through the capture of a small fraction of the mass lost by the AGB star. In narrower systems (such as the Red Rectangle) a massive circum-binary disk can form. The observations presented in this study suggest that IRAS 09425-6040 is in fact a binary system with a massive circum-binary disk. We stress that no evidence for the binarity of IRAS 09425-6040 exists yet.

\section{Conclusions}

The results of this study can be summarized as follows:

1. IRAS $09425-6040$ is a carbon star with a present-day mass-loss rate of about $210^{-6} M_{\odot} \mathrm{yr}^{-1}$ and a wind expansion velocity of $8 \mathrm{~km} \mathrm{~s}^{-1}$. The ratio of ${ }^{12} \mathrm{CO}$ to ${ }^{13} \mathrm{CO}$ derived from the millimeter pure-rotational line strength ratios suggest that this object is a J-type carbon star, similar to previously discovered silicate carbon stars;

2. The $15-45 \mu \mathrm{m}$ part of the SWS spectrum is dominated by strong emission bands from crystalline, Mg-rich silicates, that take up 60 to 80 per cent of the mass of the "small" grains;

3. The total dust mass is in the order of $210^{-3} M_{\odot}$ which is probably located in a stationary, long-lived disk, in which the grains coagulated. The high degree of crystallinity is probably the result of a low-temperature crystallization process (Molster et al. 1999a);

4. We propose that IRAS $09425-6040$ is a binary system surrounded by an oxygen-rich dust disk, and that it is the evolutionary progenitor to the Red Rectangle.

High spatial resolution mapping of this star, with infrared intereferometry, together with $2-\mathrm{D}$ radiative transfer modelling will help to reveal the spatial structure of this remarkable object. With this information we can test the above proposed (evolutionary) status and binarity of this star.

Acknowledgements. FJM acknowledges support from NWO grant 781-71-052. LBFMW and IY gratefully acknowledge financial support from an NWO "Pionier" grant.

\section{References}

Agladze, N. I., Sievers, A. J., Jones, S. A. et al. 1996, ApJ, 462,1026

Dorschner, J., Begemann, B., Henning, Th., et al. 1995, A\&A, 300,503

de Graauw, Th., Haser, L. N., Beintema, D. A., et al. 1996, A\&A, 315, L49

Groenewegen, M. A. T., van den Hoek, L. B., \& de Jong, T. 1995, A\&A, 293, 381

Jäger, C., Molster, F. J., Dorschner, J., et al. 1998, A\&A, 339, 904

Jura, M., \& Kahane, C. 1999, ApJ, 521, 302

Jura, M., Balm, S. P., \& Kahane, C. 1995, ApJ, 453, 721

Jura, M., Turner, J., \& Balm, S. P. 1997, ApJ, 474, 741

Kessler, M. F., Steinz, J. A., Anderegg, M. E., et al. 1996, A\&A, 315, L27

Knapp, G. R., \& Morris, M. 1985, ApJ, 292, 640

Koike, C., \& Shibai, H. 1998, ISAS report, No. 671

Koike, C., Tsuchiyama, A., Suto, H., et al. 1999, in Proceedings of the 32nd ISAS Lunar and Planetary Symp., 175

Lambert, D. L., Hinkle, K., \& Smith, V. V. 1990, AJ, 99, 1612 
Laor, A., \& Draine, B. T. 1993, ApJ, 402, 441

Lloyd Evans, T. 1990, MNRAS, 243, 336

Mennella, V., Brucato, J. R., Colangeli, L., et al. 1998, ApJ, 496, 1058

Molster, F. J., Yamamura, I., Waters, L. B. F. M., et al. 1999a, Nat, 401, 563

Molster, F. J., Waters, L. B. F. M., Trams, N. R., et al. 1999b, A\&A, 350, 163

Ossenkopf, V., Henning, Th., \& Mathis, J. S. 1992, A\&A, 261, 567

Osterbart, R., Langer, N., \& Weigelt, G. 1997, A\&A, 325, 609

Scott, A., Duley, W. W. 1996, ApJS, 105, 401

Waelkens, C., van Winckel, H., Waters, L. B. F. M., \& Bakker, E. J. 1996, A\&A, 314, L17
Waters, L. B. F. M., \& Molster, F. J. 1999, in Asymptotic Giant Branch Stars, Proceedings of IAU Symp. 191, ed. T. Le Bertre, A. Lèbre, \& C. Waelkens, 209

Waters, L. B. F. M., Molster, F. J., \& de Jong, T., et al. 1996, A\&A, 315, L361

Waters, L. B. F. M., Waelkens, C., \& van Winckel, H., et al. 1998, Nature, 391, 868

Willems, F. J., \& de Jong, T. 1986, ApJ, 309, L39

Yamamura, I., de Jong, T., Justtanont, K., et al. 1998, AP\&SS, 255,351

Yamamura, I., Dominik, C., de Jong, T., et al. 2000, A\&A, in press 